\title{
The Hot Winds of Novae
}

\section{PETER H. HAUSCHILDT, \\ S. STARRFIELD, ${ }^{1}$ E. BARON, ${ }^{2}$ AND F. ALLARD ${ }^{3}$}

\author{
${ }^{1}$ Department of Physics \& Astronomy, Arizona State University, Tempe, AZ 85287 \\ ${ }^{2}$ Department of Physics \& Astronomy, University of Oklahoma, Norman, OK 73019-0225 \\ ${ }^{3}$ Department of Physics, Wichita State University, Wichita, KS 67260-0032
}

We discuss the physical effects that are important for the formation of the late wind spectra of novae. Nova atmospheres are optically thick, rapidly expanding shells with almost flat density profiles, leading to geometrically very extended atmospheres. We show how the properties of nova spectra can be interpreted in terms of this basic model and discuss some important effects that influence the structure and the emitted spectrum of nova atmospheres, e.g., line blanketing, NLTE effects, and the velocity field. Most of the radiation from hot nova winds is emitted in the spectral range of the $E U V E$ satellite. Therefore, we present predicted EUVE spectra for the later stages of nova outbursts. Observations of novae with $E U V E$ could be used to test our models for the nova outburst.

\section{Introduction}

The modeling and analysis of early nova spectra has progressed significantly during the last 2-3 years by the construction of detailed model atmospheres and synthetic spectra for novae by Hauschildt et al. (1992, 1994ab, 1995) . In the early stages of the nova outburst, the spectrum is formed in an optically very thick shell (in both lines and continua) with a flat density profile, leading to very extended continuum and line forming regions (hereafter, CFR and LFR, respectively). Because of the large variation of the physical conditions inside the spectrum forming region, the classical term "photosphere" is not appropriate for novae. The large geometrical extension leads to a very large electron temperature gradient within the CFR and LFR, allowing for the observed simultaneous presence of several ionization stages of many elements. Typically, the relative geometrical extension $R_{\text {out }} / R_{\text {in }}$ of a nova atmosphere is $\approx 100 \ldots 1000$, which is much larger than the geometrical extensions of hydrostatic stellar atmospheres (even in giants $R_{\text {out }} / R_{\text {in }}$ is typically less than 2) or supernovae ( $\mathrm{SNe}$ ). Nova atmospheres are also very different from SN atmospheres with respect to their energy balance. Whereas SNe spectra show constantly decreasing color temperatures and decreasing bolometric luminosities, the color temperatures of nova atmospheres generally increase with time and their bolometric luminosity is constant. This is caused by the presence of a central energy source in novae (the hot white dwarf) which is missing in $\mathrm{SNe}$ (where the only sources of energy are the radioactive decays of the $\mathrm{Ni}$ and Co nuclei).

The electron temperatures and gas pressures typically found in nova photospheres lead to the presence of a large numbers of spectral lines, predominantly Fe-group elements, in the LFR and a corresponding influence of line blanketing on the emergent spectrum. The situation is complicated significantly by the velocity field of the expanding shell which leads to an enhancement of the overlapping of the individual lines. This in turn makes simplified approximate treatments of the radiative transfer by, e.g., the Sobolevapproximation, very inaccurate and more sophisticated radiative transfer methods, which treat the overlapping lines and continua simultaneously, must be used in order to obtain reliable models. The line blanketing also leads to strong wavelength redistribution of 


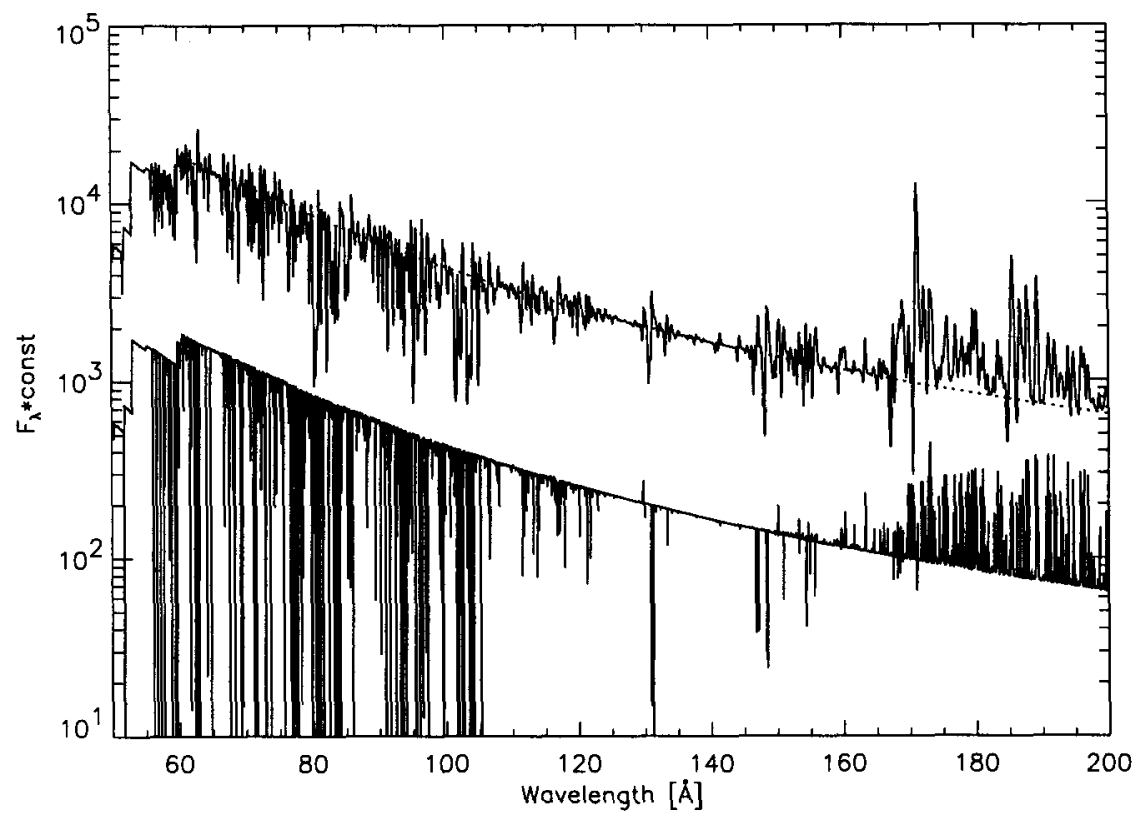

FIGURE 1. Experimental nova spectra for $T_{\text {eff }}=250000 \mathrm{~K}$ and a density law of the form $\rho \propto r^{-3}$ in the EUV spectra range. The upper spectrum is a synthetic spectrum computed with an expansion velocity of $v_{\max }=1000 \mathrm{~km} \mathrm{~s}^{-1}$. The dotted line is the corresponding continuum spectrum (all spectral lines neglected). For comparison we show in the lower spectrum (shifted by -1 dex with respect to the upper spectrum for clarity) the results for a static atmosphere with otherwise the same structure.

the radiative energy, therefore, the temperature structure of the shell must be calculated including the effects of the line blanketing.

The situation is further complicated by the fact that the (electron) densities of the CFR and LFR in a nova atmosphere are very low compared to classical stellar atmospheres. This leads to an overwhelming dominance of the radiative rates over the collisional rates. In addition, the radiation field is very non-grey. These two effects lead to large departures from local thermodynamic equilibrium (LTE) in the CFR and LFR. Therefore, NLTE effects must be included self-consistently in the model construction, in particular in the calculation of the temperature structure and the synthetic spectra. As discussed in the previous paragraph, the effects of the line blanketing on the radiative rates requires a careful treatment of the radiative transfer in the NLTE calculations and simple approximations can lead to wrong results.

In this paper, we discuss the formation of the later wind spectrum of a nova. In the later phases of the nova outburst, the spectrum of the extremely hot white dwarfs can be observed directly only in the EUV spectral range. In both the optical and UV spectral ranges the radiation from the hot wind is re-processed by the nebular around the nova. In addition, the effective temperatures of the wind are so high, in excess of $250,000 \mathrm{~K}$, that most of the energy is emitted in the EUV range. 


\section{Results}

In order to extract detailed quantitative information on velocities, densities, temperatures, and compositions, and constrain theoretical explosion models, it is necessary to model the nova spectrum in detail. We use our computer code PHOENIX (Version $5.5)$ to compute model atmospheres and synthetic spectra for novae. This is an updated version of the code used for the analyses of the early spectra of Nova Cygni 1992 (Hauschildt et al. , 1994a) and SN 1993J (Baron et al. , 1994), a more detailed description can be found in Hauschildt et al. (1995) and Allard \& Hauschildt (1995).

The low densities and complicated radiation fields in the atmosphere require that the most important species be treated using multi-level NLTE. At present, we can treat H I (15 levels), He I (11 levels), He II (15 levels), Na I (3 levels), Mg II (18 levels), Ca II (5 levels), Ne I (26 levels), and Fe II (617) in NLTE. The Fe II 617 level model atom includes 13675 primary transitions that are self-consistently included in the radiative transfer and statistical equilibrium calculations and an additional (up to) 1.2 million secondary weak transitions that are included as background line opacity (see Hauschildt \& Baron 1995 for details).

Our NLTE model atmospheres show that the early evolution of a nova shell, the "optically thick" phase, can be divided into at least 3 very different epochs. The first and very short-lived stage is the "fireball" stage, first detected in the infrared by Gehrz (see Gehrz, 1988) and analyzed in the UV by Hauschildt et al. (1994a). In this stage, the density gradient in the nova atmosphere is high, $N \approx 15$ and the effective temperatures are dropping from $\approx 15000$ to $<10000 \mathrm{~K}$ (lower $T_{\text {eff }}$ are probable but have not yet been observed). In this stage, the nova spectrum resembles that of a SN with low velocities $\left(v_{\max } \approx 4000 \mathrm{~km} \mathrm{~s}^{-1}\right.$ for Nova V1974 Cygni 1992).

As the density and temperatures of the expanding fireball drop, the material becomes optically thin and deeper layers become visible. In this stage, the "optically thick wind phase," the atmosphere evolves to a very flat density profile, $N \approx 3$, and the LFR, as well as the CFR, has a very large geometrical extension, values of $\Delta R / R \approx 100$ in the LFR are common. The large geometrical extension causes a very large temperature gradient in the LFR, typically the electron temperatures range between $1,100 \mathrm{~K}$ and $50,000 \mathrm{~K}$ for a model with $T_{\text {eff }}=6,500 \mathrm{~K}$ and from $40000 \mathrm{~K}$ to $>1,000,000 \mathrm{~K}$ for a model with $T_{\text {eff }}=200,000 \mathrm{~K}$ (we emphasize that all of the regions can be visible simultaneously in the emitted spectrum). This explains the observed fact that nova spectra can show, simultaneously, lines from different ions of the same element. The structure of the atmospheres and the calculated spectra are very sensitive to changes of the abundances of iron, carbon, nitrogen, and oxygen, which makes abundance determinations of these elements possible. However, for reliable abundance determinations, NLTE effects need to be included self-consistently for the elements under consideration. Consistent with the results of Pistinner et al. (1995) we also find that the synthetic spectra are insensitive to large changes in the luminosity. However, the spectra are very sensitive to changes in the form of the velocity profile inside the atmosphere.

In terms of the classical scheme of nova spectrum classification (McLaughlin, 1960), the increase in the effective temperature during the wind stage corresponds to the transition from the "premaximum spectrum" to the "diffuse enhanced spectrum" and later into the "nebular spectrum."

The EUV spectra of novae are dominated by the line blanketing of many thousands of overlapping spectral lines. The line blanketing changes the structure of the atmosphere, and the emitted spectrum, so radically that it must be included self-consistently in the models in order to be able to reliably compare the results to observed spectra. In Fig. 1 
we display experimental synthetic spectra for a model with $T_{\text {eff }}=250,000 \mathrm{~K}, \rho \propto r^{-3}$, and a linear velocity law with $v_{\max }=1,000 \mathrm{~km} \mathrm{~s}^{-1}$. The plot also shows the continuum spectrum obtained by neglecting all lines (dotted curve). Line blanketing has a significant impact on the spectrum emitted by the model, in particular in the EUV. Many lines in the EUV are absorption lines, only a relatively small fraction of the lines show P Cygni profiles. This is typical for nova spectra and is more pronounced in models with lower effective temperatures. The impact of the velocity field on the spectrum is demonstrated by comparing it to a spectrum emitted by a static atmosphere with otherwise identical parameters (lower spectrum in Fig. 1).

The strong coupling between continua and lines, as well as the strong overlap of lines, requires a unified treatment of the radiative transfer to (at least) full first order in $v / c$ (i.e., including advection and aberration terms, our calculations are done using a fully relativistic radiative transfer). Methods that separate continua and lines (e.g., the Sobolev approximation) give unreliable results. The "density" of lines in the EUV is so high that practically the complete wavelength range is affected by lines, even for very high effective temperatures. For slightly cooler models, most of the observed "emission lines" are just gaps between "clusters" of lines, thus they are regions of transparency which have less opacity than the surrounding wavelength regions. In addition, the radiation fields inside the nova continuum and line forming regions nowhere resemble blackbody or grey distributions. This condition, combined with the low densities, causes very large departures from LTE. The NLTE radiative transfer and rate equations must be solved self-consistently including the effects of line blanketing in the UV and optical spectral regions. The effects of neglecting the line blanketing on the departure coefficients are enormous, as shown by Hauschildt \& Ensman (1994) for the case of SN model atmospheres.

The large deviation from a blackbody energy distribution and the extreme non-gray spectrum cause a large effect of the lines on the temperature structure of nova atmospheres. The effects of line cooling (in the optically thin regions) and back-warming (in the inner regions) must be included in a proper analysis of nova spectra. The temperature changes introduced by line blanketing can amount to more than $10000 \mathrm{~K}$ in the CFR and LFR, thus changing the synthetic spectra significantly. This demonstrates that a nova model atmosphere must include line blanketing self consistently in order to derive parameters, in particular elemental abundances, otherwise the results are unreliable.

\section{Summary}

The agreement between our synthetic spectra and observed early nova spectra is very good from the UV to the near IR. We are now able to reproduce the UV and optical spectra of very different types of classical novae. The basic physics and the modeling of early nova atmospheres is now well understood but the later nova wind models are still in an experimental stage. We are currently working on improvements of the later stage models (more NLTE species). Another important step will be to investigate the effects of density inversions (clumps) on the emitted spectrum and a more systematic investigation of the effects of different velocity fields on nova spectra is also required. Furthermore, we are currently working on a detailed treatment of the "pre-nebula" phase, i.e., the phase where allowed, semi-forbidden and sometimes forbidden lines are simultaneously present in the observed nova spectra.

It is a pleasure to thank H. Störzer, J. Krautter, G. Shaviv and S. Pistinner for stimulating discussions. This work was supported in part by a NASA LTSA grant to Arizona 
State University, by NASA grant NAGW-2999; as well as grants to G. F. Fahlman and H. B. Richer from NSERC (Canada). Some of the calculations presented in this paper were performed at the San Diego Supercomputer Center (SDSC), supported by the NSF, and at the NERSC, supported by the US DoE, we thank them for a generous allocation of computer time.

\section{REFERENCES}

Allard, F. \& Hauschildt, P. H. 1995, ApJ, in press

Baron, E., Hauschimd, P. H., \& Branch, D. 1994, ApJ, 426, 334

GEHRZ, R. D. 1988, AR\&AA, 26, 377

HAUSCHILDT, P. H. \& BARON, E. 1995, JQSRT, submitted

Hauschind, P. H. \& ENSMAN, L. M. 1994, ApJ, 424, 905

Hauschildt, P. H., Starrfield, S., Austin, S. J., Wagner, R. M., Shore, S. N., \& SONNEBORN, G. 1994a, ApJ, 422, 831

Hauschildt, P. H., Starrfield, S., Shore, S. N., Allard, F., \& Baron, E. 1995, ApJ, in press

Hauschildt, P. H., Starrfield, S., Shore, S. N., Gonzales-Riestra, R., Sonneborn, G., \& Allard, F. 1994b, AJ, 108, 1008

Hauschind, P. H., Wehrse, R., Starrfield, S., \& Shaviv, G. 1992, ApJ, 393, 307

McLaughurn, D. B. 1960, in Stellar Atmospheres, edited by Greenstein, J. L., number VI in Stars and Stellar Systems, page 585, University of Chicago Press

Pistinner, S., Shaviv, G., Hauschildt, P. H., \& Starrfield, S. 1995, ApJ, in press 